\title{
Electrical memory devices based on inorganic/organic nanocomposites
}

\author{
Tae Whan Kim ${ }^{1}$, Yang Yang ${ }^{2}$, Fushan $\mathrm{Li}^{3}$ and Wei Lek Kwan ${ }^{4}$ \\ Nonvolatile memory devices based on hybrid inorganic/organic nanocomposites have emerged as excellent candidates for \\ promising applications in next-generation electronic and optoelectronic devices. Among the various types of nonvolatile memory \\ devices, organic bistable devices fabricated utilizing hybrid organic/inorganic nanocomposites have currently been receiving \\ broad attention because of their excellent performance with high-mechanical flexibility, simple fabrication and low cost. \\ The prospect of potential applications of nonvolatile memory devices fabricated utilizing hybrid nanocomposites has led to \\ substantial research and development efforts to form various kinds of nanocomposites by using various methods. Generally, \\ hybrid inorganic/organic nanocomposites are composed of organic layers containing metal nanoparticles, semiconductor \\ quantum dots (QDs), core-shell semiconductor QDs, fullerenes, carbon nanotubes, graphene molecules or graphene oxides \\ (GOs). This review article describes investigations of and developments in nonvolatile memory devices based on hybrid \\ inorganic/organic nanocomposites over the past 5 years. The device structure, fabrication and electrical characteristics of \\ nonvolatile memory devices are discussed, and the switching and carrier transport mechanisms in the hybrid nonvolatile \\ memory devices are reviewed. Furthermore, various flexible memory devices fabricated utilizing hybrid nanocomposites are \\ described and their future prospects are discussed.
}

NPG Asia Materials (2012) 4, e18; doi:10.1038/am.2012.32; published online 8 June 2012

Keywords: carrier transport; flexible; graphene; hybrid nanocomposites; nonvolatile memory; resistive switching

\section{DEVICE STRUCTURE AND FABRICATION OF THE NONVOLATILE MEMORY DEVICE}

Fabrication of nonvolatile memory devices by utilizing the evaporation method

A typical structure for a hybrid organic/inorganic nonvolatile memory device consists of composite organic molecules: metal/semiconductor nanoparticles layer sandwiched between two metal electrodes. The device area is defined by the overlap between the top and the bottom electrodes; therefore, a very high memory density can be easily achieved by using cross-bar arrays. ${ }^{1-5}$ The organic material used can consist of small organic molecules or polymers. Small organic molecules have low molecular weight and can be deposited under high vacuum without decomposition by using thermal evaporation. Polymer molecules, on the other hand, consist of much larger molecules with long chains of repeating monomer units and will decompose before evaporation begins. Polymeric materials and devices will be discussed in detail in the next section.

One advantage of thermal evaporation is that multiple layers of organic thin films can be deposited uniformly over a large area. As a result, device characteristics can be controlled by changing the materials and thickness. Ma et al. first demonstrated that resistive switching could be achieved by depositing a layer of aluminum nanoparticles between two organic layers. ${ }^{3}$ Because the depositions of the nanoparticles and the organic layer are independently controlled, the distribution of nanoparticles can be very well controlled in these devices. Figure 1 shows a typical structure for such devices. Usually, the metal cluster layer is formed by depositing a thin metal film at a very slow evaporation rate $\left(<0.1 \mathrm{As}^{-1}\right)$. The metal layer has been shown not to form a continuous film when the deposition rate and the thickness are low (Figure 1). ${ }^{6-8}$ For the case of aluminum, aluminum oxide may be formed spontaneously because of residual oxygen in the vacuum chamber. ${ }^{6}$ This insulating oxide layer is believed to be important as it can improve the charge retention properties of the nanoparticles. An oxygen plasma has also been used to form an oxide around the metal particles. ${ }^{9}$ Semiconducting materials, for example, $\mathrm{MoO}_{3}$ and so on, ${ }^{10}$ with suitable energy band levels can also be used in place of the metal particles. However, as shown by the vast differences in the electrical characteristics of the devices fabricated in different laboratories, ${ }^{3,6-8}$ the reproducibility of the device depends very much on the vacuum chamber and deposition conditions. Therefore, a more repeatable fabrication process is required for the large-scale manufacture of such devices.

\footnotetext{
1Department of Electronic Engineering, Hanyang University, Seoul, Republic of Korea; ${ }^{2}$ Department of Materials Science and Engineering, UCLA, Los Angeles, CA, USA; ${ }^{3}$ Institute of Optoelectronic Display, Fuzhou University, Fuzhou, PR China and ${ }^{4}$ School of Electrical and Electronic Engineering, Singapore Polytechnic, Singapore, Singapore Correspondence: Professor TW Kim, Department of Electronics Engineering, Hanyang University, 17 Haengdang-dong, Seongdong-gu, Seoul 133-791, Republic of Korea. E-mail: twk@hanyang.ac.kr
}

Received 17 January 2012; revised 28 March 2012; accepted 15 April 2012 


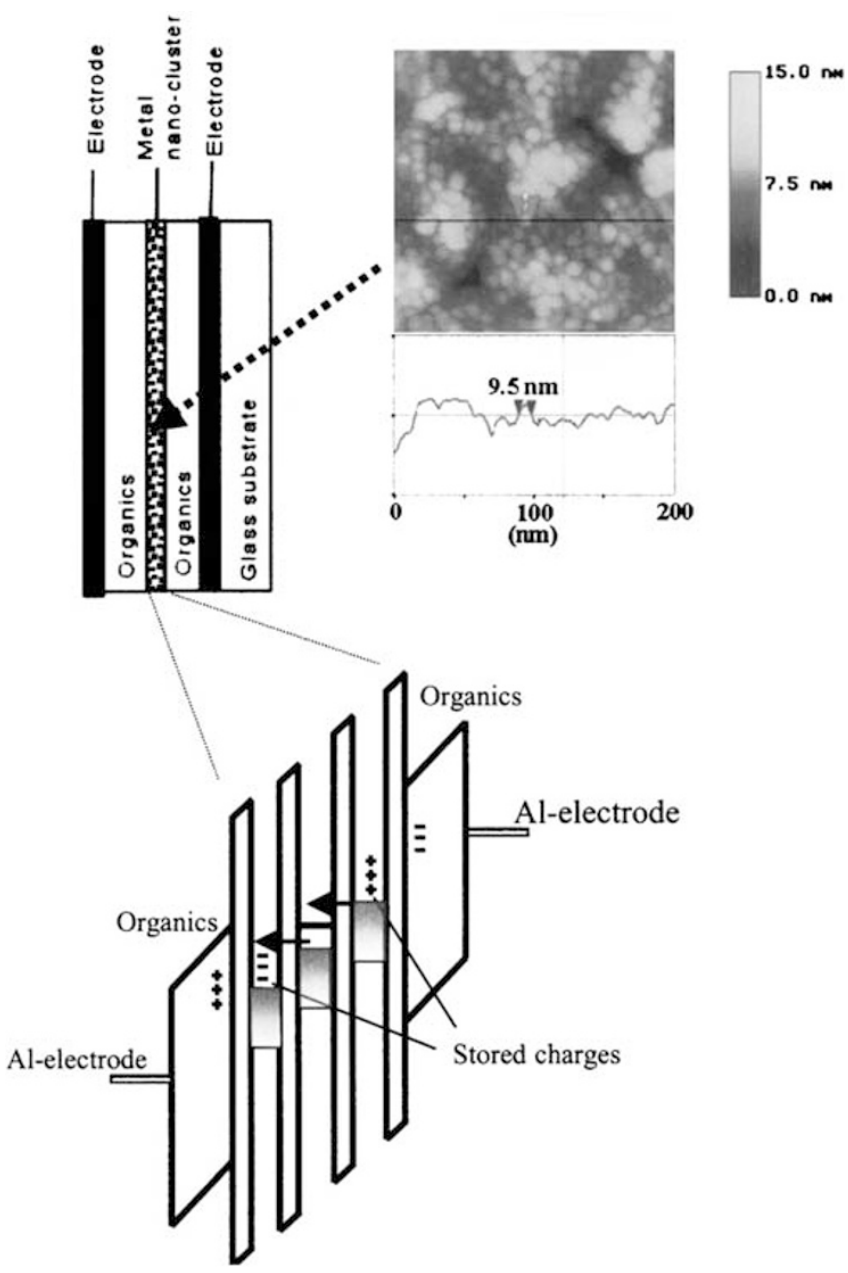

Figure 1 Typical device structure and atomic force microscope measurement of the metal cluster layer. Figure from Ma et al. ${ }^{6}$ (๔) 2003 American Institute of Physics).

Besides depositing a separate layer of metal/semiconductor clusters, metal atoms from the electrodes can also be 'driven' into the organic layer by applying a large voltage across the device during the 'forming' process. ${ }^{11}$ However, such processes are difficult to control and often result in nonuniform distributions of particles due to local field enhancements at the rough edges of the electrodes (Figure 2). ${ }^{12}$ Metals like gold can also be introduced into the organic layer during deposition. Gold, for example, is deposited at high temperature and does not react with the organic film. As a result, it readily diffuses into the organic layer, forming clusters within the organic layer. ${ }^{13}$ Care has to be taken in the fabrication of such devices so that a short circuit does not form between the top and the bottom electrodes.

\section{Fabrication of nonvolatile memory devices by using the solution} method

One of the main advantages provided by organic materials and devices is low fabrication cost and simplicity. The fabrication of these devices through thermal evaporation requires high vacuum and stringent conditions to control the morphology of the organic active layer. ${ }^{14}$ As alternatives to the more elaborate processes of vacuum evaporation and deposition of organic and inorganic molecular materials, solution processes, including spin-coating, spray-coating,
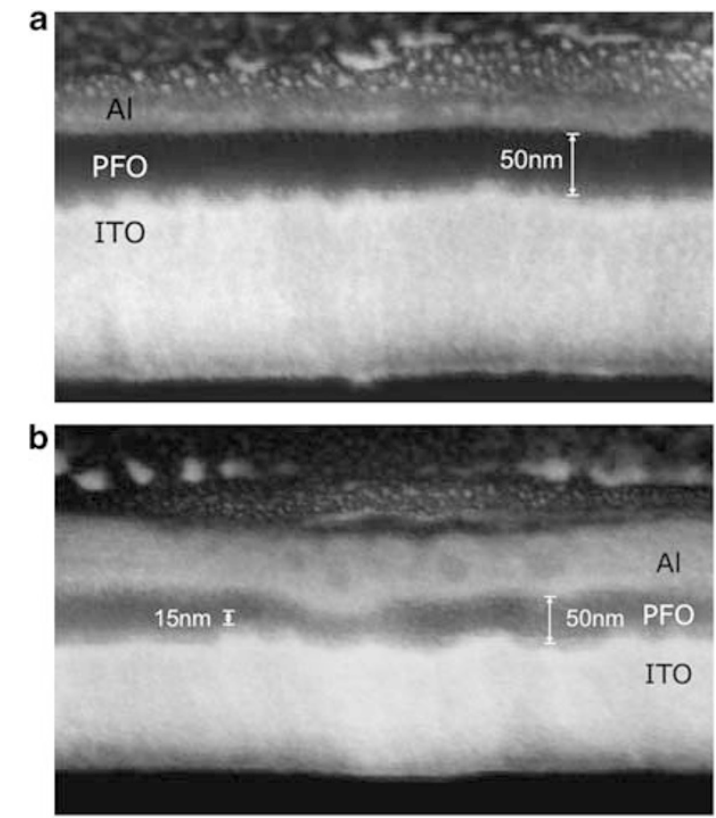

Figure 2 Cross-sectional scanning electron microscope images of the device at different spots: (a) uniform regions and (b) nonuniform regions. Figure from Lei et al. ${ }^{12}$ (๔ 2009 Elsevier).

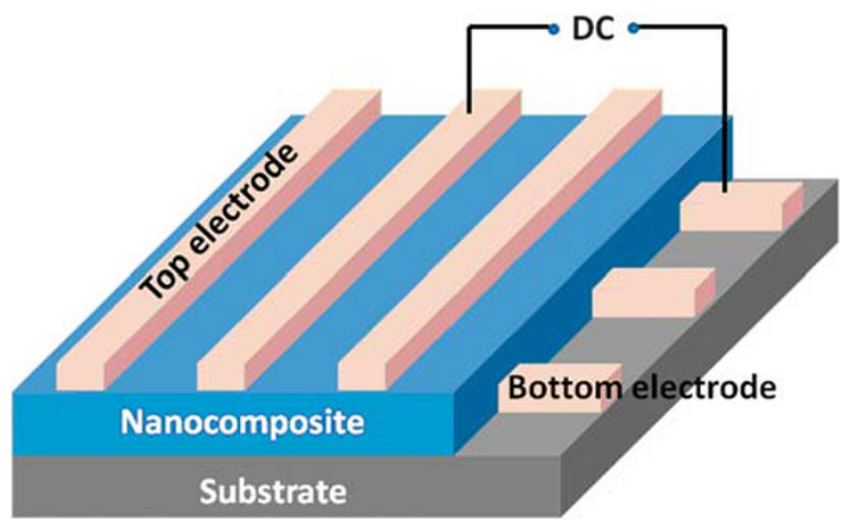

Figure 3 Schematic of a single-layer hybrid memory device.

dip-coating, roller-coating and ink-jet printing, are well known and can be used to deposit polymer materials on a variety of substrates, such as glasses, plastics, metal foils and wafers. ${ }^{15-21}$

Single-layer structure. The simplest structure for a hybrid memory device fabricated utilizing the solution method is a single-polymer layer embedded with inorganic nanomaterials and sandwiched between two metal electrodes, as shown in Figure 3. Generally, the hybrid nanocomposites are formed by dissolving inorganic nanomaterials and a polymer matrix simultaneously in a certain organic solvent with a relatively high volatility. ${ }^{22}$ To date, fabrications of single-layer-structured nonvolatile memories based on various organic/inorganic hybrid nanocomposites have been reported. Inorganic species used in these memories include semiconductor nanoparticles ( $\mathrm{ZnO}, \mathrm{CdSe}, \mathrm{Si}, \mathrm{CuO}$ and so on) and metal nanoparticles $\left(\mathrm{Au}, \mathrm{Ag}, \mathrm{FeNi}\right.$ and so on). ${ }^{22-28}$ Both insulating polymers, such as polyimide (PI), poly(methylmethacrylate) and polystyrene, and conducting polymers, such as poly $(N$ vinylcarbazole) and poly(2-methoxy-5-(2-ethyhexoxy)-1,4-phenylene 
vinylene), are used as a matrix for the inorganic nanoparticles. ${ }^{29-33}$ Most of these hybrid thin films are formed on a certain substrate by using a spin-coating technique, where the inorganic nanoparticles are randomly dispersed in the polymer layer. The morphology and uniformity of the hybrid films can be controlled by varying the precursor concentration and spin conditions.

Multilayer structure. The single-layer structure fabricated via the solution process offers the simplest way to realize a nonvolatile memory device. However, several key issues need to be addressed for this type of memory device. One of the problems that single-layer memories face is the cross talk of neighboring memory cells due to the presence of parasitic conduction paths, which causes misreading during the operating process. ${ }^{34}$ A possible solution to this problem is to use one diode and one resistor or one transistor and one resistor structures to construct an individual memory cell with high reading accessibility. ${ }^{35,36}$ Therefore, multilayer structures might be required to realize one diode and one resistor or one transistor and one resistor cells.

On the other hand, device reproducibility may not be ensured because of the contact uncertainty at the interface between the hybrid layer and the metal electrode. Thus, the multilayer structure, where inorganic nanoparticles are sandwiched between organic layers, has been employed in order to obtain memories with high stability and reproducibility. ${ }^{37}$ The main challenge to obtain such a structure is the dissolving of the underlying polymer layer caused by the solvent used to form the upper polymer layer. Thus, the key point in realizing a multilayer structure is the availability of an appropriate polymer material. It has been shown that a polymer thin film made of PI, after annealing at a certain temperature, can well withstand the organic solvent used to form the polymer layer. Some works concerning the formation of multilayer-structured nonvolatile memory devices by using PI materials have been reported, and their memory effects have been demonstrated as well. ${ }^{38-40}$ It was shown that the cell reproducibility and the cell-to-cell uniformity of the hybrid memory devices could be significantly improved by employing a multilayer structure.

An ultra-high storage density, which can be achieved by using a vertically stacked three-dimensional architecture, has been shown to be required for next-generation nonvolatile memory devices. ${ }^{41-43}$ Therefore, a multilayer structure based on hybrid nanocomposites may offer a promising approach to future ultra-high-density memories. Recently, Song et al. ${ }^{43}$ developed a hybrid resistive memory device with a three-dimensional stacked $8 \times 8$ cross-bar array by using a composite of PI and 6-phenyl-C61 butyric acid methyl ester. The results indicated that the memory cells in each layer could be programmed or erased with a uniform parameter. That study provided a simple and straightforward method for increasing the storage density without using a complicated downscaling procedure.

\section{Fabrication of nonvolatile memory devices utilizing graphene materials embedded in a polymer matrix}

Graphene, comprised of one or several monolayers of carbon atoms packed into a two-dimensional honeycomb lattice, is predicted to have remarkable properties, such as superior mobility, thermal conductivity, current-carrying capability and room-temperature ballistic transport, which make it an attractive material for electric, optoelectronic and photonic devices. ${ }^{44-51}$ The two-dimensionality of graphene allows scaling beyond the conventional semiconductor technology, which could be instrumental for high-density storage- memory applications. Furthermore, if graphene is integrated with polymers and flexible substrates, a spectrum of graphene-based memory applications, including transparent, flexible, and wearable electronics, is possible. ${ }^{52}$ Some works concerning graphene-based memory devices have been reported recently. ${ }^{53-58}$ For instance, Jeong et al. ${ }^{54}$ reported a reliable and reproducible bipolar resistive-switching effect in a GO film spin-casted on a flexible substrate, as shown in Figure 4. The device exhibited a long retention time and a low switching voltage, but the ON/OFF current ratio was relatively modest, $\sim 100$, while $10^{5}-10^{6}$ has been typically achieved in complementary metal-oxide semiconductor devices.

Graphene sheets, acting as charging and discharging media, have been particularly interesting because of their being promising candidates for hybrid-nanocomposite-based resistive-switching devices for potential applications in low-cost flexible memories. However, the pristine graphene materials are unsuitable for intercalation in large species, such as polymer chains, because graphene has a pronounced tendency to agglomerate in a polymer matrix, which leads to difficulty in material processing and to a high cost of device fabrication. One of the approaches for improving the solubility of the graphene material is to modify GO by using a chemical method. ${ }^{59-61}$ For example, Zhuang et al. ${ }^{62}$ demonstrated a single-layer hybrid bistable memory containing GO sheets covalently grafted with soluble conjugated polymer triphenylamine-based polyazomethine (TPAPAM). The device was fabricated by using a simple spincoating technique, and the TPAPAM-GO-based memory device exhibited typical bistable electrical switching and a nonvolatile rewritable memory effect, with a turn-on voltage of about $-1 \mathrm{~V}$ and an ON/OFF current ratio of about $10^{3}$. This is thought to be the first example of a polymer-modified graphene-based memory device.

However, multilayer-structured memory devices still need to be developed in order to improve device reproducibility. A nonvolatile hybrid bistable device fabricated with graphene sandwiched between two insulating poly(methylmethacrylate) polymer layers was reported by Son et al. ${ }^{63}$ An ON/OFF ratio of $4.4 \times 10^{6}$ was maintained for retention times longer than $10^{5} \mathrm{~s}$, and the endurance number of the ON/OFF switching was $1.5 \times 10^{5}$ cycles. The bistable behavior for the poly(methylmethacrylate)-graphene-based device was attributed to conducting filaments having been formed in the hybrid active layer. $\mathrm{Wu}$ et al. ${ }^{64}$ demonstrated a nonvolatile hybrid memory device employing a tri-layer structure, PI/PI:GO/PI, in which insulating polymer PI was used as the matrix for the GO sheets. Apart from the high ON/OFF ratio $\left(\sim 10^{5}\right)$ and the long retention time $(>1400 \mathrm{~s})$, the device exhibited excellent cell-to-cell uniformity (70 out of 81 cells, 86\%), as shown in Figure 5.

As a whole, recent results clearly show that graphene/polymer hybrid nanocomposites are able to present a superior memory effect with a large ON/OFF ratio, good endurance, long retention time and high cell-to-cell uniformity. These advantages may enable graphene/ polymer nanocomposites to serve as a storage media in low-cost flexible nonvolatile memories. However, for practical applications, some issues, for example, the cross talk between the adjacent memory cells, the difficulty of in-situ synthesis of graphene in a polymer matrix and so on, still need to be addressed. These problems may be overcome in the near future as more and more research is focused on graphene-based hybrid memory devices.

\section{ELECTRICAL CHARACTERISTICS OF THE HYBRID NONVOLATILE MEMORY DEVICE}

In general, the electrical properties of nonvolatile memories based on hybrid nanocomposites can be investigated by using current-voltage 
a
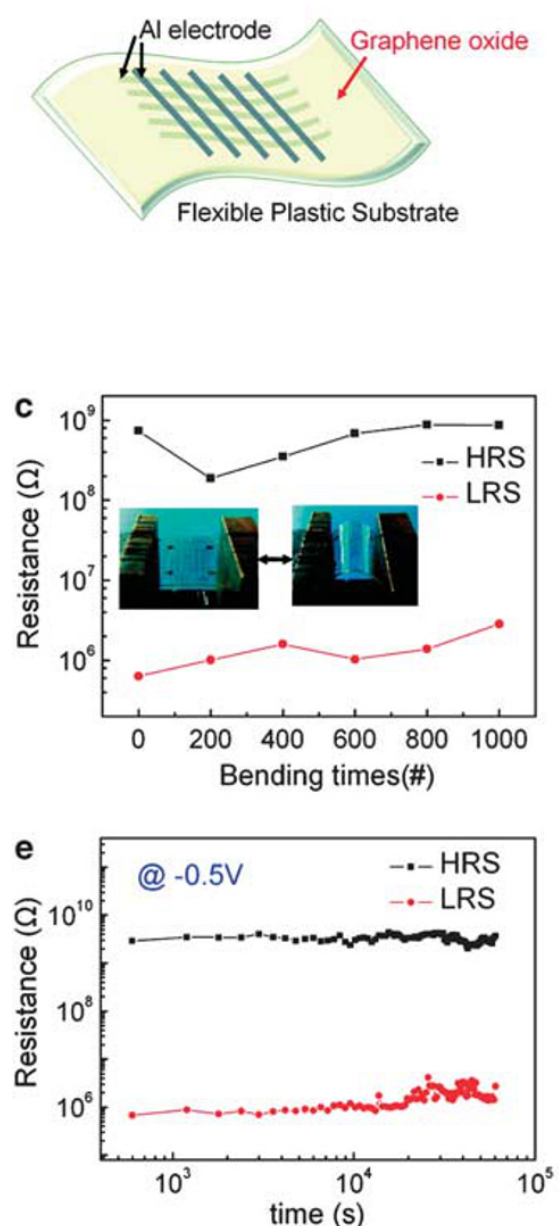
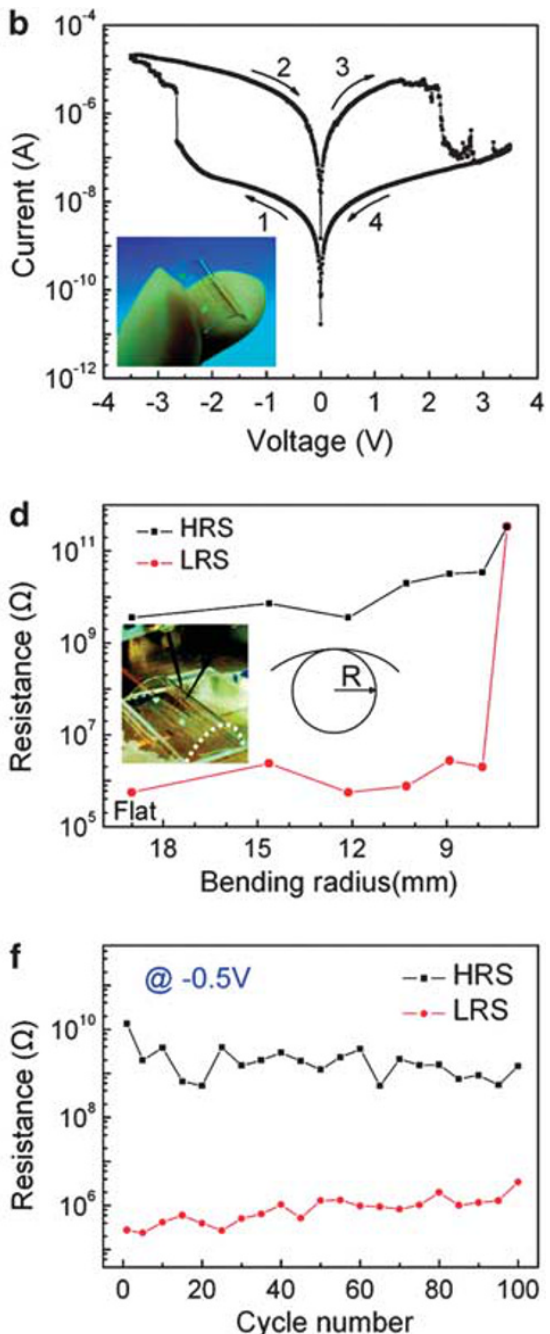

Figure 4 (a) Schematic of a GO-based flexible cross-bar memory device. (b) Typical I-V curve of an AI/GO/AI/PES device plotted on a semilogarithmic scale. The arrows indicate the voltage sweep direction. The left inset is a real photo image of a device. (c) Continuous bending effect of an Al/GO/Al/PES device. The insets show photographs of two repeated bending states. (d) The resistance ratio between the high-resistance state and the low-resistance state as a function of the bending radius $(R)$. The inset is a photograph of an I-V measurement being performed under a flexed condition. (e) Retention test of an $\mathrm{Al} / \mathrm{GO} / \mathrm{Al} / \mathrm{PES}$ device read at $-0.5 \mathrm{~V}$. (f) Endurance performance of an Al/GO/Al/PES device measured during 100 sweep cycles. Figure from Jeong et al.54 (C 2010 American Chemical Society).

(I-V) measurements. The applied voltage across the device is varied in a cycle from negative bias to positive bias and then to negative bias again. Two distinct conducting states, the ON state and the OFF state, corresponding to the high-current and the low-current states, respectively, can be observed in the I-V curves. The electrical hysteresis behavior shown in the I-V characteristics is an essential feature of a resistive-switching memory device. ${ }^{65}$ The resistive transition $(\mathrm{OFF} \rightarrow \mathrm{ON})$ occurs at a certain applied voltage, which is defined as the turn-on voltage. The transition is equivalent to the 'writing' process in a digital memory cell. A high turn-on voltage means high power consumption and always reduces the reliability. Thus, the operating voltage for a hybrid nonvolatile memory should be only a few volts. After the transition is finished, the ON state remains in the device even after the power is turned off, which reveals the nonvolatile nature of the hybrid memory device. ${ }^{6}$

Depending on the I-V characteristics, three types of nonvolatile memory effects, write-once-read-many-times and unipolar and bipolar electrical switching, can be observed for hybrid devices. The writeonce-read-many-time memory exhibits an irreversible electrical transition upon external voltage stimulation. Both unipolar and bipolar memories can restore their original conducting state when a voltage with the same (for unipolar) or different (for bipolar) polarity is applied to the device. Typically, the maximum ON/OFF ratio in the $\mathrm{I}-\mathrm{V}$ curves for hybrid nonvolatile memories is $>10^{3}$, which distinguishes the two states and decreases the probability of misreading.

In the case of a nonvolatile memory based on hybrid nanocomposites, the memory effect is strongly correlated with the presence of inorganic nanomaterials, for example, $\mathrm{ZnO}$ QDs, $\mathrm{Au}$ nanoparticles, CdSe nanoparticles and carbon nanotubes, embedded in a polymer matrix because the current difference between the ON and the OFF states for the polymer-only device is negligible. ${ }^{66}$ Recently, for a hybrid device containing Au QDs embedded in a PI layer, Wu et al. ${ }^{40}$ reported an electrical bistability with an ON/OFF ratio of $\sim 10^{8}$, which is one of the largest values for a stable hybrid memory device. In the case of a unipolar nonvolatile memory, due to the presence of a negative differential resistance in the I-V characteristics, the OFF state can be regained when a forward bias is given to the memory device. ${ }^{67-70}$ For bipolar memories, the OFF state of the hybrid 
a
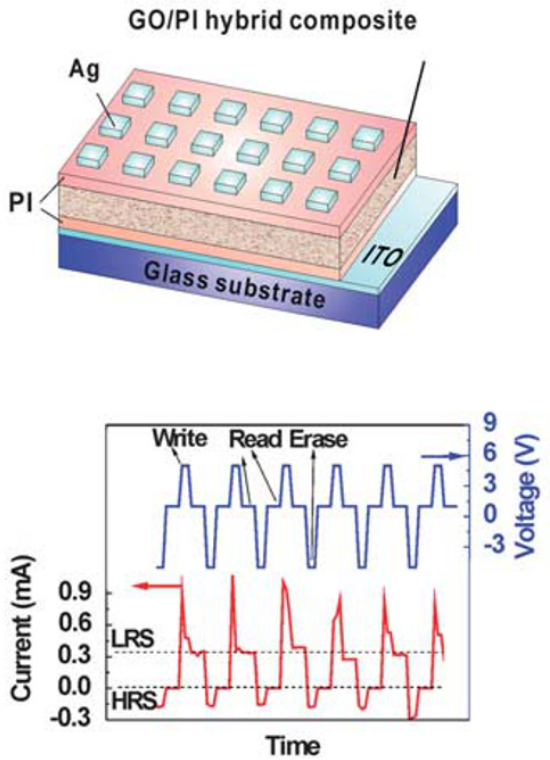

e

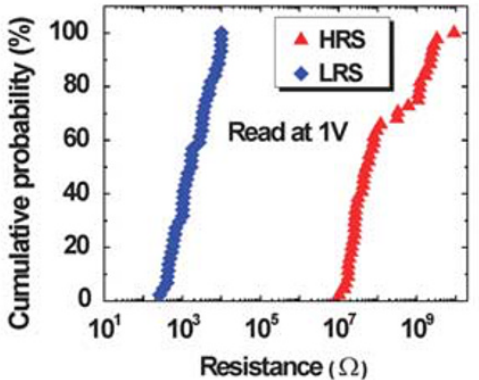

b

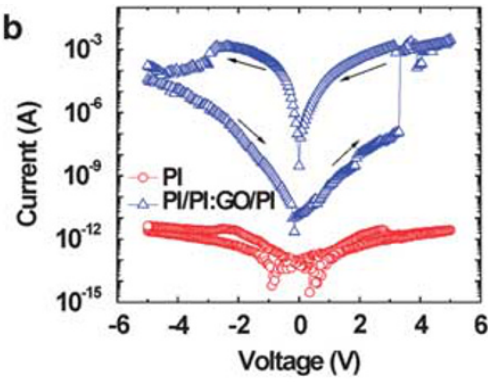

d
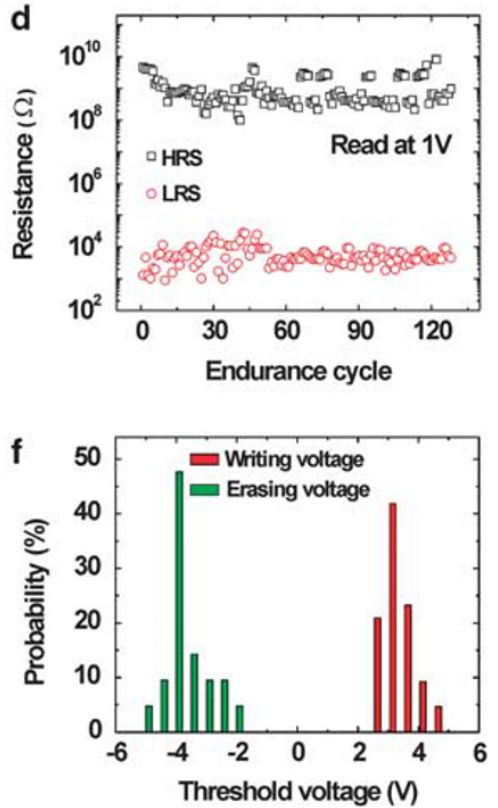

Figure 5 (a) Schematic of the hybrid memory device containing GO sheets embedded in a $\mathrm{PI}$ layer. (b) $\mathrm{I}-\mathrm{V}$ characteristics for Ag/PI/GO:PI/PI/indium tin oxide (ITO) and Ag/PI/ITO devices. (c) Write-read-erase-read sequence test for a Ag/PI/GO:PI/PI/ITO memory device. (d) Resistances of the low-resistance state and high-resistance stateat $1 \mathrm{~V}$ for 130 cycles. (e) Cumulative probability data for the resistances of the low-resistance state and high-resistance state. (f) Threshold voltage distributions for the 'write' and the 'erase' processes. Figure from Wu et al. ${ }^{64}$ (๑ 2011 American Institute of Physics).

device can be recovered by applying a reverse bias voltage, which is equivalent to the 'erasing' process in a digital memory cell.

For a nonvolatile rewritable hybrid memory, a 'write-read-eraseread' sequence test is commonly carried out to investigate the endurance of the device. Before the $\mathrm{ON}$ and OFF states are no longer distinguishable, the number of cycles should be large enough to obtain an advantage over conventional semiconductor-based memories, which show a typical endurance of $10^{3}-10^{7}$ cycles under ambient conditions. Besides the endurance, the retention time is also an important parameter for a memory device. A test for the retention time can be performed by keeping the hybrid device in the ON or OFF state and observing the device current over time to identify the conductive state of the device. The retention time clearly reflects the environmental stability and capability of the memory to maintain its storage. Most commercial products claim to have retention times of $>10$ years. ${ }^{71}$ As reported by Son et al., ${ }^{63}$ some hybrid nonvolatile memories, in which a hybrid nanocomposite of graphene-poly(methylmethacrylate) was used as an active layer (Figure 6), have been demonstrated to exhibit retention time comparable to those of commercial products.

The switching speed is defined as the shortest time for programming or erasing a hybrid memory cell. Obviously, for practical applications, a high switching speed $(<100 \mathrm{~ns})$ is necessary to meet the requirement of ultra-high-density information storage. Ma et al. ${ }^{3}$ reported that a hybrid memory cell could exhibit a switching speed as fast as $10 \mathrm{~ns}$.

The device yield for hybrid memories should definitely be taken into account for future commercialization. Unfortunately, the yield is generally rather low due to the uncontrollability of the microstructure of the hybrid active layers. The uncertainty at the hybrid layer/ electrode interface also contributes to low device reproducibility and cell-to-cell uniformity. Thus, the morphology of the hybrid active film should be precisely controlled because the carrier transport of a hybrid memory device is strongly governed by the microstructure of the hybrid film. The growth parameters, such as the concentration of inorganic nanoparticles, should be carefully adjusted to obtain a repeatable nonvolatile memory effect. It has been shown that the ON/OFF ratio of a hybrid memory based on a CdSe QD-poly $(N-$ vinylcarbazole) structure can be maximized when the content of CdSe QDs is optimized. ${ }^{72}$ Excessive CdSe leads to an aggregation of the poly $(N$-vinylcarbazole) matrix and to a degradation of the memory properties, including the device reproducibility.

\section{SWITCHING AND CARRIER TRANSPORT MECHANISMS IN THE HYBRID NONVOLATILE MEMORY DEVICE}

The switching mechanism has been under debate since the discovery of the memory phenomenon in hybrid devices. It has been argued 
a
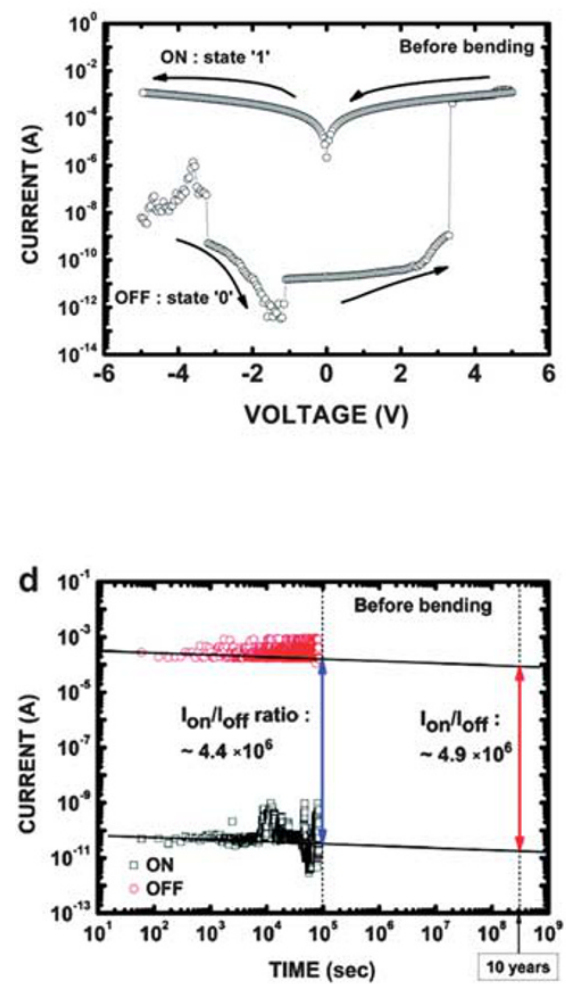

b
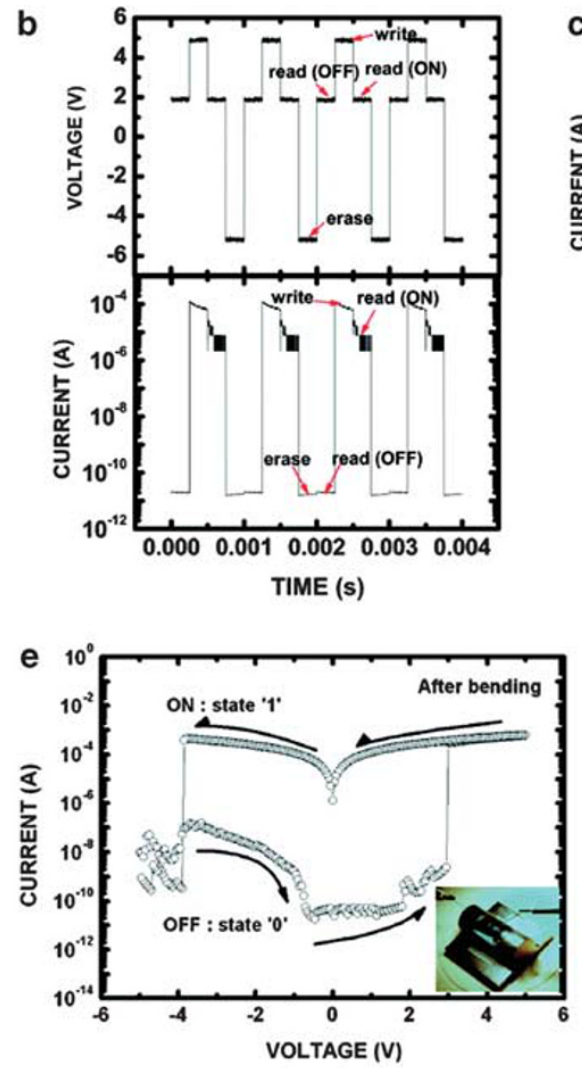
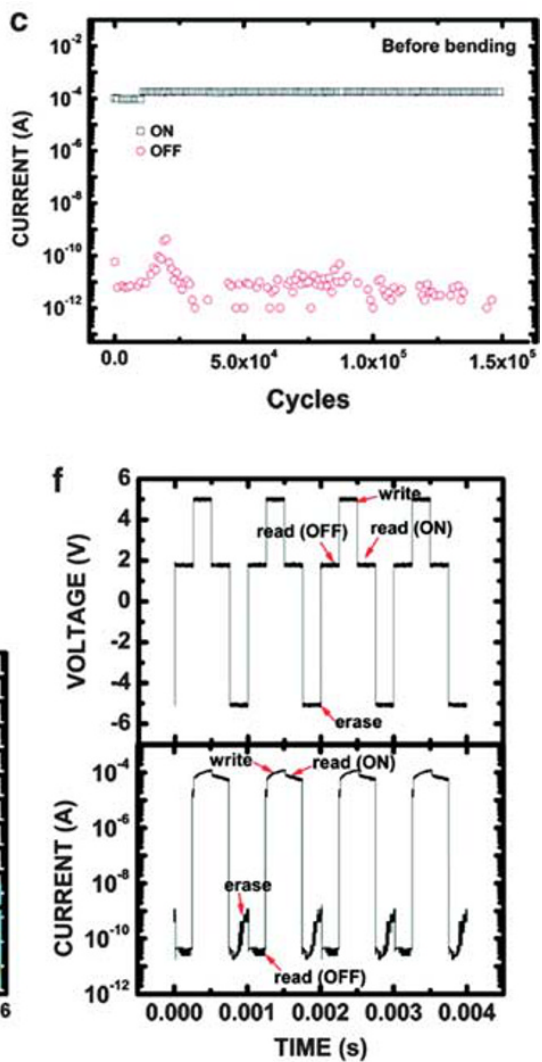
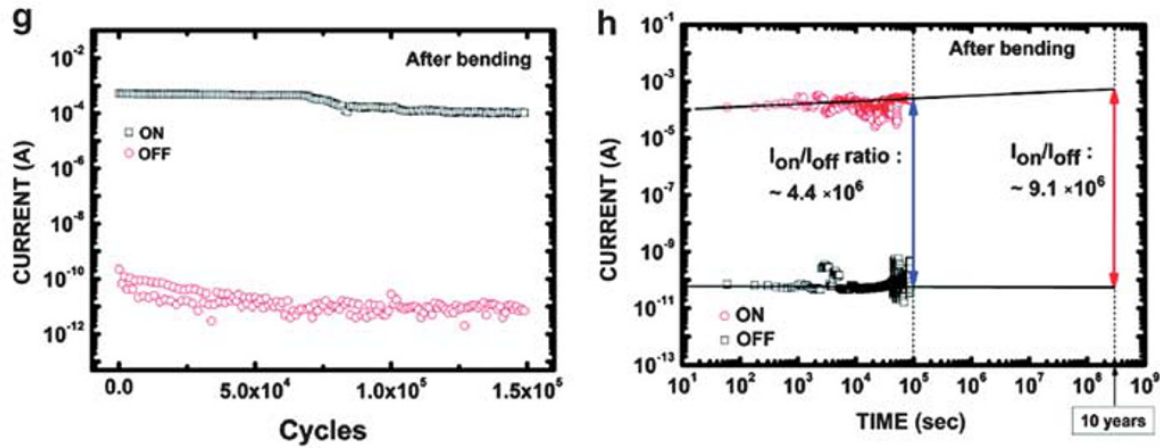

Figure 6 (1) Memory characterization of the organic bistable devices (OBDs) before bending: (a) I-V curves for the Al/poly(methylmethacrylate) (PMMA)/ graphene/PMMA/ITO/polyethylene terephthlate (PET) device, (b) operation of a write-read-erase-read (5/2/-5/2 V) sequence, (c) cycling stress test of the Al/ PMMA/graphene/PMMA/ITO/PET device under a stress voltage of $2 \mathrm{~V}$ per $4 \mathrm{~ms}$ and (d) retention test of OBDs in an ambient environment. (2) Memory characterization after bending (bending radius, $10 \mathrm{~mm}$ ): (e) I-V curves for the Al/PMMA/graphene/PMMA/ITO/PET devices after bending, (f) operation of a write-read-erase-read (5/1.8/-5/1.8V) sequence, $(\mathbf{g})$ cycling stress test of the Al/PMMA/graphene/PMMA/ITO/PET device under a stress voltage of $1.8 \mathrm{~V}$ per $4 \mathrm{~ms}$ and (h) retention test of the memory device in an ambient environment. Figure from Son et al. ${ }^{63}$ (๑ 2010 American Chemical Society).

that because the device characteristics are so similar across different material systems, one underlying mechanism might explain the phenomenon completely. However, a unifying explanation has been elusive due to inconclusive evidence. The lack of understanding of the switching mechanism has hindered progress towards the commercialization of these devices.

One approach to understanding the switching mechanism is to study the carrier transport mechanisms of the device at the two resistive states. Fittings to the $\mathrm{I}-\mathrm{V}$ curves and their temperature dependences are often used to study the charge transport during device operation. Ohmic conduction, thermionic emission, spacecharge-limited current (SPLC) and Fowler-Nordheim tunneling are few of the more common mechanisms used to explain charge transport in organic devices. Even though this is not always explicitly acknowledged, the current distribution is almost always assumed to be uniformly distributed across the entire device. However, in reality, this is often difficult to achieve because of localized nonuniformities in the devices. As a result, the parallel pathways with different transport mechanisms may exist in one device, which may complicate analysis.

More recently, high-resolution transmission electron microscopy has been used to examine the change in the nanoscale structure of a device before and after switching. This may provide an important new evidence for a complete understanding of the switching phenomenon. In this section, recent works on the switching and the charge carrier 
transport mechanisms in inorganic/organic nanocomposite memories are reviewed.

\section{Filament formation}

One of the earliest explanations for the large change in resistance is the formation of the highly conductive pathways in the composite layer. As in copper-ion memories, ${ }^{73-75}$ mobile metallic ions from the electrodes or the nanoparticles can migrate to form a conductive filament between the two electrodes when a high enough voltage is applied to the device. It has been observed using a current-sensing atomic force microscope ${ }^{76-79}$ and an infrared microscope ${ }^{80}$ that only the switching occurred in localized areas of the devices. Highly conductive spots are created after the device is switched to the low conductive state. In some cases, damage to the electrodes due to highcurrent densities at such spots is detected. ${ }^{81}$ Because the filament is metallic in nature, the temperature dependence of the current at the low conductive state is very low.

Because of the simplicity of the idea, many groups have attributed the switching phenomenon to the formation and the destruction of the conductive pathways despite of the lack of direct evidence. As the filaments are usually nanoscale and sparsely distributed, probing the filament directly is very difficult. Only recently has direct observation of the filaments been possible, through the use of high-resolution transmission electron microscopy, for carefully prepared cross-sections of the device. ${ }^{11,82}$ Figure 7 shows that gold atoms from the electrode are injected into the polymer film after a high voltage is applied to the device ('forming' process). Figures $8 \mathrm{a}$ and $\mathrm{b}$ show a highly conductive pathway between the top and the bottom electrodes after the device is switched to a low conductive state. The change in the temperature dependence of the current of the device (Figure 8c) clearly shows that the device has changed from an electronic conduction mechanism to a filamentary one after the switching process. Although a device that switches with a filamentary mechanism has been shown to be able to switch more than a few thousand cycles, ${ }^{83}$ this is undesirable as it requires a large switching current and may damage the electrodes.

\section{SPLC}

Usually a non-ohmic contact is formed between the electrode and the organic/inorganic hybrid layer. When that happens, the current that
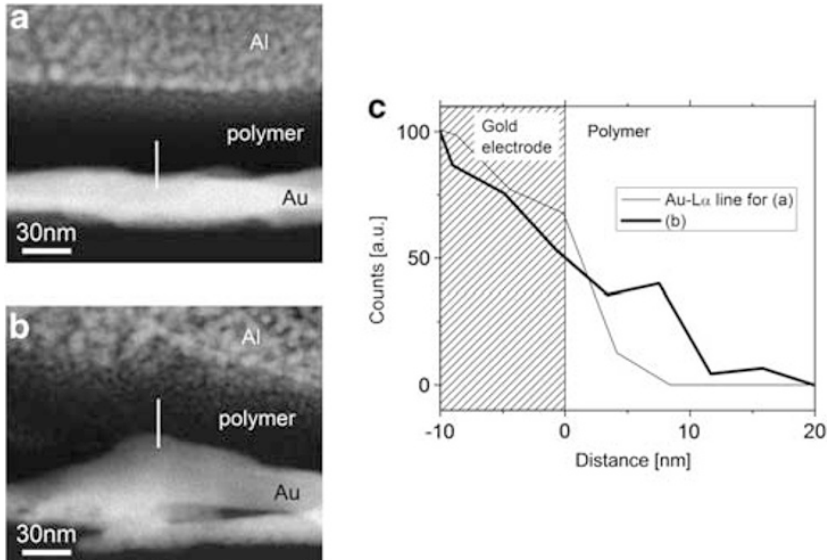

Figure 7 Cross-sectional scanning transmission electron microscope (STEM) images of devices whose bottom gold electrode was (a) flat and (b) irregular. The white lines denote the locations of the TEM-energy-dispersive X-ray spectroscopy (EDS) line scan. (c) TEM-EDS line scan data for (a) and (b). Figure from Kwan et al. ${ }^{11}$ (๑ 2009 American Institute of Physics). flows through the device is injection limited. The I-V and temperature characteristics will, thus, reflect the injection mechanism of the interface. The two most common injection mechanisms found in organic devices are thermionic emission and Fowler-Nordheim tunneling. ${ }^{84,85}$ During the switching process, as more carriers are injected into the composites, traps that are formed from nanoparticles are hypothesized to fill up, which eventually causes the current to increase rapidly, reaching a SPLC. ${ }^{86}$ The nanoparticles can act as traps as long as the energy level is within the lowest unoccupied molecular orbital and highest occupied molecular orbital levels of the organic matrix. Figure 9 shows a transition from thermionic emission to a SPLC when the bias voltage is increased. Interestingly, the trap is able to hold the charges very stably, with the retention time being extrapolated to as long as 10 years. ${ }^{87}$

\section{Simmons and Verderder's model}

In the Simmons and Verderder's model, when the traps are empty, the device is in the low resistive state, which is contrary to that of the SPLC model. The Simmons and Verderder's model argues that when the trap is not filled, the impurity band of the composite layer is free to conduct current. However, as more charges are introduced into the material through a larger bias voltage, the charges start to occupy the impurity states, thus lowering the impurity band. This prevents additional charge from being injected into the device. The trapped charge can be removed by applying an even larger voltage bias to the device. ${ }^{88,89}$ This model is successful in explaining the negative differential resistance, whereby the current decreases with increasing voltage bias.
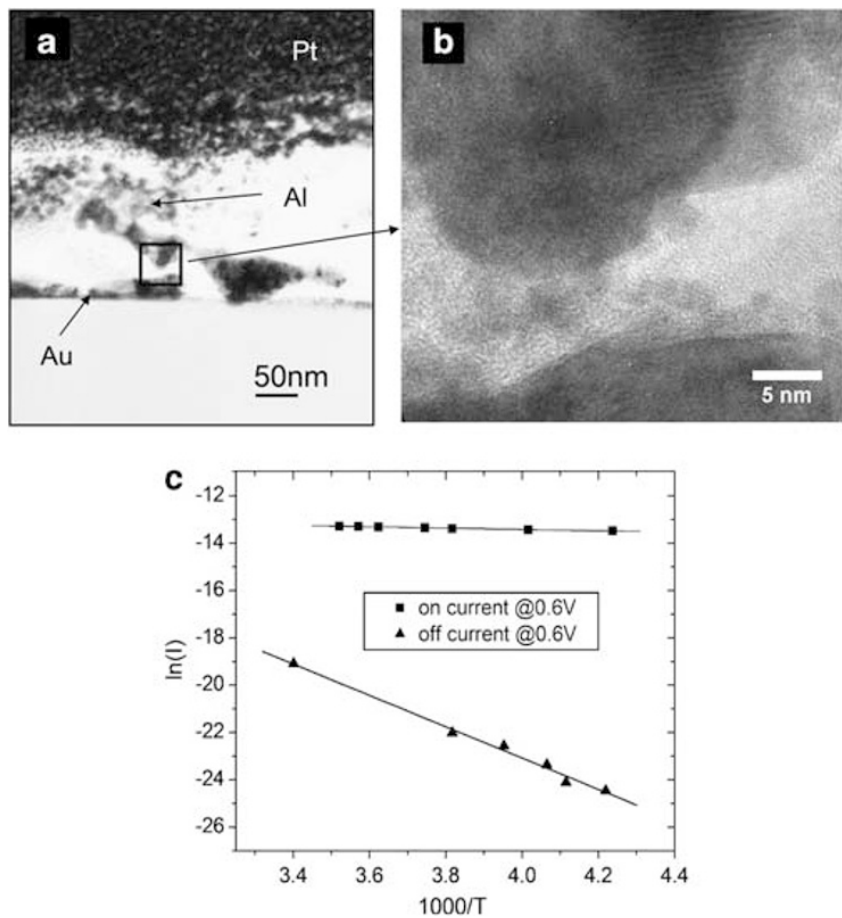

Figure 8 (a) TEM cross-sectional view of the device near the filament. Near the edge of the gold electrode, the top electrode was almost in contact with the bottom electrode. (b) Close-up TEM image of the boxed region in (a). (c) Arrhenius plots of the currents measured at $0.6 \mathrm{~V}$ at the $\mathrm{ON}$ and OFF states of the device. Figure from Kwan et al. ${ }^{11}$ (๔ 2009 American Institute of Physics). 


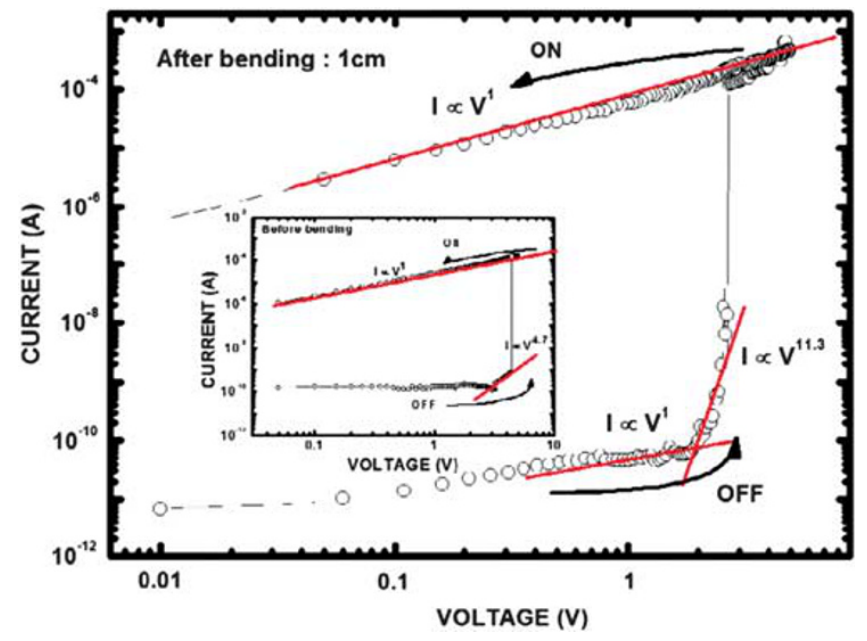

Figure $9 \mathrm{~A}$ log-log plot of the current as a function of the applied voltage for Al/PMMA/UGS/PMMA/ITO/PET devices. The curves are fitted for the SCLC mechanism. The inset presents a log-log plot of the current as a function of the voltage for the bistable organic memory devices before bending. Figure from Son et al. ${ }^{87}$ (๑ 2011 IOP Publishing Ltd.).

\section{Electric-field-induced charge transfer}

Because the nanocomposite layer is usually less than a few hundred nanometers, the electric field across the device can be very large, even though the applied bias is only a few volts. This high field is thought to cause charges to be transferred between donors and acceptors in the composite layer. Depending on the relative energy levels, the organic matrix and metallic/semiconducting nanoparticles can act as donors and acceptors, respectively. The metal particles are usually surrounded by an insulating layer, so the charges are prevented from moving back even when the applied voltage is removed. As the electrons from the organic matrix are transferred from the highest occupied molecular orbital level to the nanoparticles, additional holes are created after the charge transfer, thereby increasing the conductivity of the device..$^{90}$

In a competing theory on electric field transfer of charge, Ling et al. ${ }^{91}$ suggest that a charge-transfer complex, which may be insulating or conducting, ${ }^{92}$ is formed after the threshold electric field has been reached. In the case of an electron-donating polymer and a GO composite, the electron from the polymer is transferred to the giant $\pi$-conjugated system of the GO, thereby reducing the insulating GO to a highly conductive graphene. This substantially enhances the conductivity. ${ }^{62}$

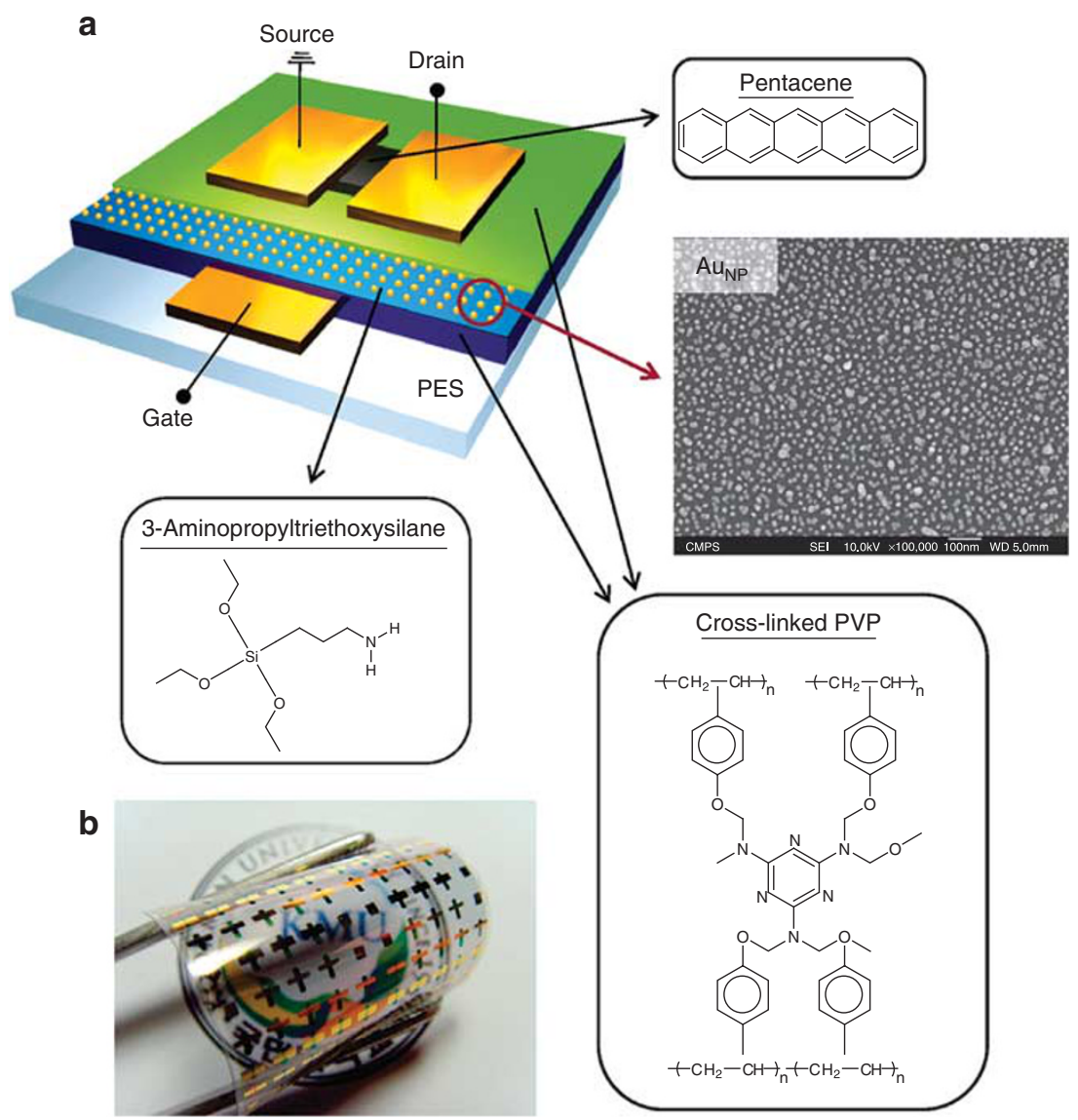

Figure 10 (a) Three-dimensional schematic diagram of the flexible hybrid memory device architecture. Patterned Ti/Au on a PES plastic substrate was used as the gate electrode, and cross-linked PVP layers were used as the blocking and the tunneling organic dielectric layers. The self-assembled gold nanoparticle charge storage layers were formed on the APTES-coated PVP blocking dielectric layer. A pentacene active layer and gold source/ drain contacts were formed to make the organic transistor-based memory devices. The chemical structures of the organic layers are shown in the figure. (b) Photograph of a fabricated flexible organic memory device. (The device size is $3 \times 3 \mathrm{~cm}^{2}$.) Figure from Kim and Lee ${ }^{93}$ (๑ 2010 American Chemical Society). 


\section{FLEXIBLE MEMORY DEVICE FABRICATED UTILIZING HYBRID} NANOCOMPOSITES

One of the main advantages of a nonvolatile memory device based on hybrid nanocomposites is the possibility of realizing flexible memories at extremely low fabrication cost. These hybrid devices can be processed at low temperatures and, therefore, are regarded as one of the most important types of information-storage components for future stretchable/portable electronic device applications. ${ }^{2,93-96}$ For example, Kim and Lee ${ }^{93}$ developed nonvolatile memory devices on plastic substrates (PES) based on a pentacene layer embedded with self-assembled gold nanoparticles, as shown in Figure 10. The nanofloating gate memory exhibited a large memory window that could be maintained for $>1$ year. The hybrid memory was reliable after several hundreds of repeated programming/erasing operation cycles. Additionally, the mechanical flexibility was confirmed using the bending-cycle test over 1000 times. Nonvolatile electrical bistability of a hybrid device fabricated utilizing CdSe/ZnS nanoparticles embedded in a conducting poly $(N$-vinylcarbazole $)$ polymer layer on flexible polyvinylidene difluoride and polyethylene terephthlate substrates has also been reported. ${ }^{96}$ The results indicate that the hybrid bistable memory devices are prospects for potential applications in flexible nonvolatile flash memory devices. ${ }^{96}$

Electrical stability of a hybrid memory during bending is important for practical applications in nonvolatile information storage. The memory should be reliable after thousands of bending-cycle tests. However, frequent folding of the memory device could cause physical and electrical damage at the conducting substrate. Metals and indium tin oxide are widely used as electrode materials for hybrid memory devices. ${ }^{5,97-99}$ However, their brittle properties under bending may become obstacles for application in flexible devices. For this reason, Ji et al. ${ }^{100}$ developed a flexible hybrid resistive memory device with transparent multilayer graphene electrodes on a polyethylene terephthlate substrate (Figure 11). The active layer of the memory is a hybrid nanocomposite of PI and 6-phenyl-C61 butyric acid methyl ester. The memory devices show typical write-once-readmany-time characteristics with an ON/OFF ratio of over $\sim 10^{6}$. The current levels can be maintained upon bending for up to 10000 times. A stainless-steel foil can also serve as a conducting substrate for a nonvolatile memory device because of its high conductivity and flexibility. Lee et al. ${ }^{101}$ reported the resistive-switching characteristics of $\mathrm{ZnO}$ thin films grown on stainless steel for flexible nonvolatile memory devices. The device performance was not degraded upon bending, which indicates high potential for flexible memory applications. However, the introduction of a stainless-steel substrate may lead to an enhanced fabrication cost for commercialized memory devices. In addition, a stainless-steel substrate might not be appropriate for flexible memory devices that require high transparency.

\section{CONCLUSIONS AND FUTURE PROSPECTS}

Hybrid inorganic/organic nanocomposites provide a frontier for unique functions with promising applications in nonvolatile memory devices. Organic bistable devices fabricated utilizing nanocomposites have emerged as excellent candidates for potential applications in the next-generation nonvolatile memory devices. In this article, the current bistabilities, operating mechanisms, memory mechanisms and carrier transport mechanisms of organic bistable devices fabricated utilizing nanocomposites were described on the basis of recently published experimental data.

Currently, nonvolatile memories based on hybrid nanocomposites still have some problems. In comparison with Si-based memories, the

\section{Graphene electrodes Organic layer/Al electrodes}

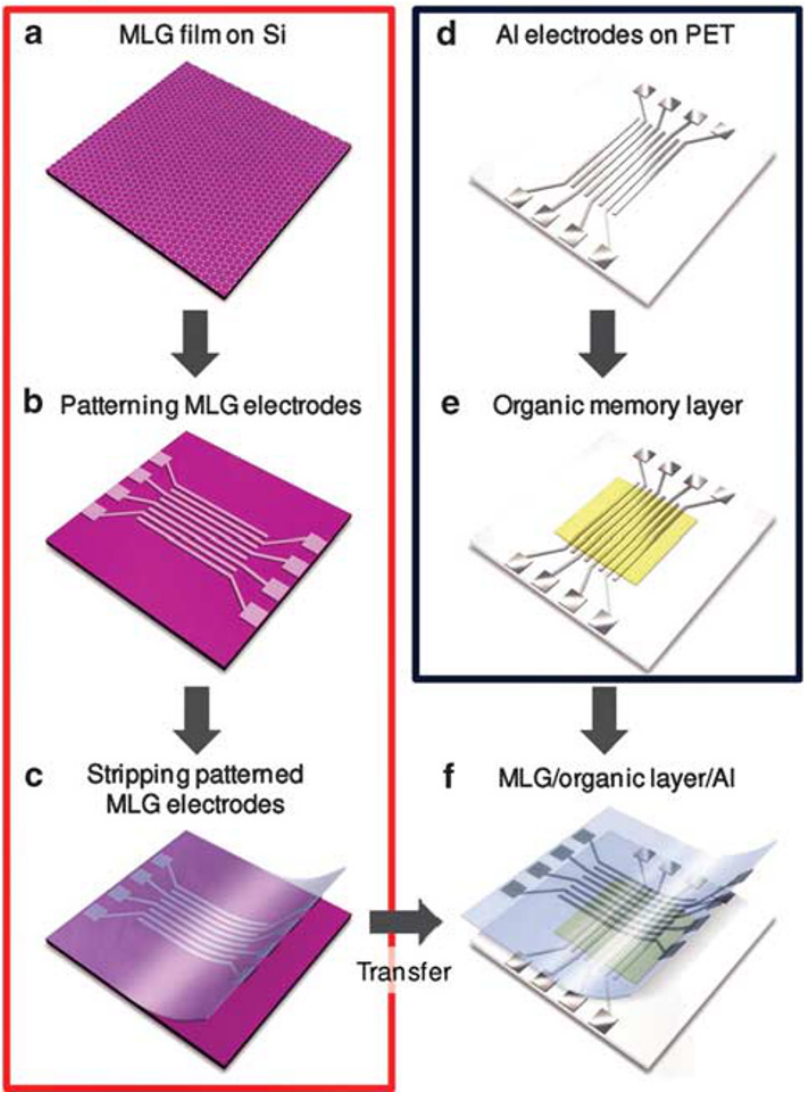

Figure 11 Schematic representation showing the fabrication process for the hybrid flexible memory device with transparent graphene top electrodes. Figure from Ji et al. ${ }^{100}$ (๔ 2011 American Chemical Society).

switching speed of hybrid memories should be further improved, even though some hybrid systems have exhibited exceptional switching times of $\sim 10 \mathrm{~ns}$, because most hybrid memories have typical switching speeds in the millisecond range. Other problems that nonvolatile hybrid memories face are device-to-device uniformity and cell reproducibility. Solution processing not only simplifies the fabrication of nonvolatile hybrid memories but also leads to a high uncertainty in the film's microstructure. Thus, for practical applications, hybrid memories should have a high device yield comparable to that of Si-based memories. Obviously, the memory properties of hybrid devices should be improved, so new hybrid materials with superior memory effects should be explored. Moreover, the carrier transport mechanisms in hybrid nonvolatile memories are still under debate and should be clarified in the near future.

One of the important applications of nonvolatile memory devices fabricated utilizing nanocomposites is flexible memory device, which is necessary for fascinating applications of nonvolatile memory devices in portable equipment. The device performance should be reproducible, regardless of repetitive bending. Because scaled-down experiments of memory devices with nanoscale electrodes are very important for enhancing storage density, the inherent problems due to the limitations of organic lithography for fabrication of organic bistable devices with nanoscale electrodes based on inorganic/organic nanocomposites should be overcome. As an alternative, a nonvolatile memory with a three-dimensional architecture should be developed for the realization of an ultra-high-density memory based on hybrid 
nanocomposites. As a whole, nonvolatile memories based on inorganic/organic nanocomposites hold promise for potential applications in the next-generation portable nonvolatile memory devices.

\section{ACKNOWLEDGEMENTS}

This work was supported by the National Research Foundation of Korea (NRF) grant funded by the Korea government (MEST) (no. 2010-0018877).

1 Lu, W. \& Lieber, C. M. Nanoelectronics from the bottom up. Nat. Mater. 6, 841-850 (2007)

2 Sekitani, T., Yokota, T., Zschieschang, U., Klauk, H., Bauer, S., Takeuchi, K., Takamiya, M., Sakurai, T. \& Someya, T. Organic nonvolatile memory transistors for flexible sensor arrays. Science 326, 1516-1519 (2009).

3 Ma, L. P., Liu, J. \& Yang, Y. Organic electrical bistable devices and rewritable memory cells. Appl. Phys. Lett. 80, 2997-2999 (2002).

4 Lee, K. H., Lee, G., Lee, K., Oh, M. S., Im, S. \& Yoon, S. M. High-mobility nonvolatile memory thin-film transistors with a ferroelectric polymer interfacing $\mathrm{ZnO}$ and pentacene channels. Adv. Mater. 21, 4287-4291 (2009).

5 Kuang, Y., Huang, R., Tang, Y., Ding, W., Zhang, L. \& Wang, Y. Flexible singlecomponent-polymer resistive memory for ultrafast and highly compatible nonvolatile memory applications. IEEE Elec. Dev. Lett. 31, 758-760 (2010).

6 Ma, L., Pyo, S., Ouyang, J., Xu, Q. \& Yang, Y. Nonvolatile electrical bistability of organic/metal-nanocluster/organic system. Appl. Phys. Lett. 82, 1419-1421 (2003).

7 Bozano, L. D., Kean, B. W., Deline, V. R., Salem, J. R. \& Scott, J. C. Mechanism for bistability in organic memory elements. Appl. Phys. Lett. 84, 607-609 (2004).

8 Bozano, L., Kean, B., Beinhoff, M., Carter, K., Rice, P. \& Scott, J. Organic materials and thin-film structures for cross-point memory cells based on trapping in metallic nanoparticles. Adv. Funct. Mater. 15, 1933-1939 (2005).

9 Park, J. G., Nam, W. S., Seo, S. H., Kim, Y. G., Oh, Y. H., Lee, G. S. \& Paik, U. G. Multilevel nonvolatile small-molecule memory cell embedded with Ni nanocrystals surrounded by a NiO tunneling barrier. Nano Lett. 9, 1713-1719 (2009).

10 Chang, T-Y., Cheng, Y-W. \& Lee, P-T. Electrical characteristics of an organic bistable device using an Al/Alq/nanostructured MoO/Alq/p-Si structure. Appl. Phys. Lett. 96, 043309-043311 (2010).

11 Kwan, W. L., Lei, B., Shao, Y. Prikhodko, S. V., Bodzin, N. \& Yang, Y. Direct observation of localized conduction pathways in photocross-linkable polymer memory. J. Appl. Phys. 105, 124516-124521 (2009).

12 Lei, B., Kwan, W. L., Shao, Y. \& Yang, Y. Statistical characterization of the memory effect in polyfluorene based non-volatile resistive memory devices. Org. Electron. 10, 1048-1053 (2009).

13 Aristov, V. Y., Molodtsova, O. V., Laubschat, C., Zhilin, V. M., Aristova, I. M., Kveder, V. V. \& Knupfer, M. Properties of hybrid organic-inorganic systems: Au nanoparticles embedded into an organic CuPc matrix. Appl. Phys. Lett. 97, 113103-113106 (2010)

14 Li, F., Son, D. Seo, S., Cha, H., Kim, H., Kim, B., Jung, J. H. \& Kim, T. W. Organic bistable devices based on core/shell CdSe/ZnS nanoparticles embedded in a conducting poly(N-vinylcarbazole) polymer layer. Appl. Phys. Lett. 91 122111-122114 (2007).

15 Lin, Q., Liaw, D., Teo, E. Y., Zhu, C., Chan, D. S., Kang, E. \& Neoh, K. Polyme memories: bistable electrical switching and device performance. Polymer 48, 5182-5201 (2007).

16 Azarova, N. A., Owen, J. W., McLellan, C. A., Grimminger, M. A., Chapman, E. K., Anthony, J. E. \& Jurchescu, O. D. Fabrication of organic thin-film transistors by spraydeposition for low-cost, large-area electronics. Org. Electron. 11, 1960-1965 (2010).

17 Kim, D., Jeong, S., Shin, H., Xia, Y. \& Moon, J. Heterogeneous interfacial properties of ink-jet-printed silver nanoparticulate electrode and organic semiconductor. Adv. Mater. 20, 3084-3089 (2008).

18 Andersen, T. R., Larsen-Olsen, T. T., Andreasen, B., Bottiger, A. P. L, Carle, J. E., Helgesen, M., Bundgaard, E., Norrman, K., Andreasen, J. W., Jorgensen, M. \& Krebs, F. C. Aqueous processing of low-band-gap polymer solar cells using roll-to-roll methods. ACS Nano 5, 4188-4196 (2011).

19 Zhou, Y., He, Q., Yang, Y., Zhong, H., He, C., Sang, G., Liu, W., Yang, C., Bai, F. \& Li, $Y$. Binaphthyl-containing green- and red-emitting molecules for solution-processable organic light-emitting diodes. Adv. Funct. Mater. 18, 3299-3306 (2008).

20 Chen, F., Wu, J., Lee, C., Huang, W., Chen, H. P. \& Chen, W. Flexible polyme photovoltaic devices prepared with inverted structures on metal foils. IEEE Elec. Dev. Lett. 30, 727-729 (2009).

21 Gelinck, G., Heremans, P., Nomoto, K. \& Anthopoulos, T. D. Organic transistors in optical displays and microelectronic applications. Adv. Mater. 22, 3778-3798 (2010).

$22 \mathrm{Li}$, F., Kim, T. W., Dong, W. \& Kim, Y. Formation and electrical bistability properties of $\mathrm{ZnO}$ nanoparticles embedded in polyimide nanocomposites sandwiched between two C60 layers. Appl. Phys. Lett. 92, 011906-011909 (2008).

23 Portney, N. G., Martinez-Morales, A. A. \& Ozkan, M. Nanoscale memory characterization of virus-templated semiconducting quantum dots. ACS Nano 2, 191-196 (2008)
24 Kolliopoulou, S., Dimitrakis, P., Normand, P., Zhang, H. L., Cant, N., Evans, S. D. Paul, S., Pearson, C., Molloy, A., Petty, M. C. \& Tsoukalas, D. Hybrid silicon-organic nanoparticle memory device. J. Appl. Phys. 94, 5234-5240 (2003).

25 Jung, J. H., Kim, J., Kim, T. W., Song, M. S., Kim, Y. H. \& Jin, S. Nonvolatile organic bistable devices fabricated utilizing $\mathrm{Cu}_{2} \mathrm{O}$ nanocrystals embedded in a polyimide layer. Appl. Phys. Lett. 89, 122110-122113 (2006).

26 Leong, W. L., Mathews, N., Mhaisalkar, S., Lam, Y. M., Chen, T. P. \& Lee, P. S. Micellar poly(styrene-b-4-vinylpyridine)-nanoparticle hybrid system for non-volatile organic transistor memory. J. Mater. Chem. 19, 7354-7361 (2009).

27 Kim, W. T., Jung, J. H., Kim, T. W. \& Son, D. I. Current bistability and carrier transport mechanisms of organic bistable devices based on hybrid Ag nanoparticle-polymethy methacrylate polymer nanocomposites. Appl. Phys. Lett. 96, 253301-253303 (2010).

28 Kim, J. H., Jin, J. Y., Jung, J. H., Lee, I. T., Kim, W., Lim, S. K., Yoon, C. S. \& Kim, Y. $\mathrm{H}$. Formation and electrical properties of $\mathrm{Ni}_{1-x} \mathrm{Fe}_{\mathrm{x}}$ nanocrystals embedded in a polyimide layers for applications as nonvolatile flash memories. Appl. Phys. Lett. 86, 032904-032907 (2005).

29 Huang, C., Chen, J., Hsieh, C., Song, H., Wang, Y., Horng, L., Tian, C. \& Hwang, S. Stability in the memory state of the silica nanoparticle-doped hybrid aligned nematic device. J. Appl. Phys. 109, $023505-023509$ (2011)

30 Mabrook, M. F., Pearson, C., Kolb, D., Zeze, D. A. \& Petty, M. C. Memory effects in hybrid silicon-metallic nanoparticle-organic thin film structures. Org. Electron. 9 816-820 (2008).

31 Lee, S. H., Yun, D. Y., Jung, J. H., You, J. H., Kim, T. W., Ryu, E. \& Kim, S. W. Variations in the memory capability of nonvolatile memory devices fabricated using hybrid composites of InP nanoparticles and a polystyrene layer due to the scale-down. J. Nanosci. Nanotechnol. 11, 449-452 (2011).

32 Lai, P. Y. \& Chen, J. S. Electrical bistability and charge transport behavior in Au nanoparticle/poly(N-vinylcarbazole) hybrid memory devices. Appl. Phys. Lett 93, 153305-153308 (2008).

33 Li, F., Son, D., Cha, H., Seo, S., Kim, B., Kim, H., Jung, J. \& Kim, T. W. Memory effect of CdSe/ZnS nanoparticles embedded in a conducting poly[2-methoxy-5(2-ethylhexyloxy)-1,4-phenylene-vinylene] polymer layer. Appl. Phys. Lett. 90, 222109-222112 (2007).

34 Cho, B., Song, S., Ji, Y., Kim, T. \& Lee, T. Organic resistive memory devices: performance enhancement, integration, and advanced architectures. Adv. Funct. Mater. 21, 2806-2809 (2011).

35 Kim, T. W., Choi, H., Oh, S. H., Wang, G., Kim, D. Y., Hwang, H. \& Lee, T. One transistor-one resistor devices for polymer non-volatile memory applications. Adv. Mater. 21, 2497-2500 (2009).

36 Teo, E. Y. H., Zhang, C., Lim, S. L., Kang, E., Chan, D. S. H. \& Zhu, C. An organicbased diode-memory device with rectifying property for crossbar memory array applications. IEEE Elec. Dev. Lett. 30, 487-489 (2009).

37 Li, F. Son, D., Ham, J., Kim, B., Jung, J. H. \& Kim, T. W. Memory effect of nonvolatile bistable devices based on CdSe/ZnS nanoparticles sandwiched between C60 layers. Appl. Phys. Lett. 91, 162109-162112 (2007).

$38 \mathrm{Kim}, \mathrm{H}$., Cho, W., Lee, D. U., Kim, S. P. \& Kim, E. K. Improvement of charge storage characteristics on floating gated nonvolatile memory devices with $\ln _{2} \mathrm{O}_{3}$ nanoparticles embedded polyimide gate insulator. Appl. Phys. Lett. 91, 043513-043516 (2007).

39 Kim, J. H., Baek, K. H., Kim, C. K., Kim, Y. B. \& Yoon, C. S. Formation of gold nanoparticles embedded in a polyimide film for nanofloating gate memory. Appl. Phys. Lett. 90, 123118-123121 (2007).

40 Wu, C., Li, F., Guo, T., Qu, B., Chen, Z. \& Gong, Q. Efficient nonvolatile rewritable memories based on three-dimensionally confined Au quantum dots embedded in ultrathin polyimide layers. Jpn. J. Appl. Phys. 50, 030204-030207 (2011).

41 Yun, J., Cho, S. \& Park, B. Stacked-nanowire device with virtual source/drain (SD VSD) for 3D NAND flash memory application. Solid-State Elec. 64, 42-46 (2011).

42 Kim, J., Hong, A. J., Kim, S. M., Shin, K., Song, E. B., Hwang, Y., Xiu, F., Galatsis, K., Chui, C. O., Candler, R. N., Choi, S., Moon, J. \& Wang, K. L. A stacked memory device on logic 3D technology for ultra-high-density data storage. Nanotechnology 22 254006-254009 (2011)

43 Song, S., Cho, B., Kim, T., Ji, Y., Jo, M., Wang, G., Choe, M., Kahng, Y. H., Hwang, H. \& Lee, T. Three-dimensional integration of organic resistive memory devices. Adv. Mater. 22, 5048-5052 (2010)

44 Geim, A. K. \& Novoselov, K. S. The rise of graphene. Nat. Mater. 6, 183-191 (2007)

45 Bonaccorso, F., Sun, Z., Hasan, T. \& Ferrari, A. C. Graphene photonics and optoelectronics. Nat. Photonics 4, 611-622 (2010).

46 Rana, F. Graphene optoelectronics: plasmons get tuned up. Nat. Nanotechnol. 6, 611-612 (2011).

47 Balandin, A. A. Toward ubiquitous environmental gas sensors-capitalizing on the promise of graphene. Nat. Mater. 10, 569-581 (2011).

48 Zhu, Y., Murali, S., Cai, W., Li, X., Suk, J. W., Potts, J. R. \& Ruoff, R. S. Graphene and graphene oxide: synthesis, properties, and applications. Adv. Mater. 22, 3906-3924 (2010)

49 Schwierz, F. Graphene transistors. Nat. Nanotechnol. 5, 487-496 (2010).

50 Abergel, D. S. L., Apalkov, V., Berashevich, J., Ziegler, K. \& Chakraborty, T. Properties of graphene: a theoretical perspective. Adv. Phys. 59, 261-482 (2010).

51 Allen, M. J., Tung, V. C. \& Kaner, R. B. Honeycomb carbon: a review of graphene. Chem. Rev. 110, 132-145 (2010).

52 Wang, D., Li, F., Zhao, J., Ren, W., Chen, Z., Tan, J., Wu, Z., Gentle, I., Lu, G. Q. \& Cheng, H Fabrication of graphene/polyaniline composite paper via in situ anodic electropolymerization for high-performance flexible electrode. ACS Nano 3, 17451752 (2009). 
53 Zhan, N., Olmedo, M., Wang, G. \& Liu, J. Graphene based nickel nanocrystal flash memory. Appl. Phys. Lett. 99, 113112-113115 (2011).

54 Jeong, H. Y., Kim, J. Y., Kim, J. W., Hwang, J. O., Kim, J., Lee, J. Y., Yoon, T. H., Cho, B. J., Kim, S. O., Ruoff, R. S. \& Choi, S. Graphene oxide thin films for flexible nonvolatile memory applications. Nano Lett. 10, 4381-4386 (2010).

55 Wu, C., Li, F., Zhang, Y. \& Guo, T. Recoverable electrical transition in a single graphene sheet for application in nonvolatile memories. Appl. Phys. Lett. 100, 042105-042109 (2012)

56 Gunlycke, D., Areshkin, D. A., Li, J., Mintmire, J. W. \& White, C. T. Graphene nanostrip digital memory device. Nano Lett. 7, 3608-3611 (2007).

57 Stuetzel, E. U., Burghard, M., Kern, K., Traversi, F., Nichele, F. \& Sordan, R. Graphene nanoribbon memory cell. Small 6, 2822-2825 (2010).

58 Hong, S. K., Kim, J. E., Kim, S. O., Choi, S. \& Cho, B. J. Flexible resistive switching memory device based on graphene oxide. IEEE Elec. Dev. Lett. 31, 1005-1007 (2010).

59 Hummers, W. \& Offeman, R. Preparation of graphitic oxide. J. Am. Chem. Soc. 80, 1339-1339 (1958).

60 Stankovich, S., Dikin, D. A. Dommett, G. H. B., Kohlhaas, K. M., Zimney, E. J., Stach, E. A., Piner, R. D., Nguyen, S. T. \& Ruoff, R. S. Graphene-based composite materials. Nature 442, 282-286 (2006).

61 Kuilla, T., Bhadra, S., Yao, D., Kim, N. H., Bose, S. \& Lee, J. H. Progress in polymer science. Prog. Polym. Sci. 35, 1350-1375 (2010).

62 Zhuang, X., Chen, Y., Liu, G., Li, P., Zhu, C., Kang, E., Noeh, K., Zhang, B., Zhu, J. \& $\mathrm{Li}, \mathrm{Y}$. Conjugated-polymer-functionalized graphene oxide: Synthesis and nonvolatile rewritable memory effect. Adv. Mater. 22, 1731-1735 (2010).

63 Son, D. I., Kim, T. W., Shim, J. H., Jung, J. H., Lee, D. U., Lee, J. M., Park, W. I. \& Choi, W. K. Flexible organic bistable devices based on graphene embedded in an insulating poly(methyl methacrylate) polymer layer. Nano Lett. 10, 2441-2447 (2010).

64 Wu, C., Li, F., Zhang, Y., Guo, T. \& Chen, T. Highly reproducible memory effect of organic multilevel resistive-switch device utilizing graphene oxide sheets/polyimide hybrid nanocomposite. Appl. Phys. Lett. 99, 042108-042111 (2011).

65 Yang, Y., Ouyang, J., Ma, L., Tseng, R. J. \& Chu, C. Electrical switching and bistability in organic/polymeric thin films and memory devices. Adv. Funct. Mater. 16, 1001-1014 (2006)

66 Li, F., Son, D. I., Kim, B. J. \& Kim, T. W. Nonvolatile electrical bistability and operating mechanism of memory devices based on CdSe/ZnS nanoparticle/polymer hybrid composites. Appl. Phys. Lett. 93, 021913-021916 (2008).

67 Vilkman, M., Solehmainen, K., Laiho, A., Sandberg, H. G. O. \& Ikkala, O. Negative differential resistance in polymeric memory devices containing disordered block copolymers with semiconducting block. Org. Electron. 10, 1478-1482 (2009).

68 Chen, J., Xu, L., Lin, J., Geng, Y., Wang, L. \& Ma, D. Negative differential resistance effect in organic devices based on an anthracene derivative. Appl. Phys. Lett. 89, 083514-083517 (2006)

69 Yu, L. \& Lee, C. Investigation of three-terminal organic-based devices with memory effect and negative differential resistance. Appl. Phys. Lett. 95, 103305-103308 (2009).

70 Xie, X. N., Wang, J., Loh, K. P. \& Wee, A. T. S. Aggregates-induced dynamic negative differential resistance in conducting organic films. Appl. Phys. Lett. 95, 203302-203305 (2009)

71 Lee, M., Lee, C. B., Lee, D., Lee, S. R., Chang, M., Hur, J. H., Kim, Y., Kim, C., Seo, D. H., Seo, S., Chung, U., Yoo, I. \& Kim, K. A fast, high-endurance and scalable nonvolatile memory device made from asymmetric $\mathrm{Ta}_{2} \mathrm{O}_{5-\mathrm{x}} / \mathrm{TaO}_{2-\mathrm{x}}$ bilayer structures. Nat. Mater. 10, 625-630 (2011).

$72 \mathrm{Li}, \mathrm{F}$. , Cha, H., Seo, S., Son, D., Kim, H. \& Kim, T. W. Investigation of carrier transport mechanism in CdSe/PVK hybrid nanocomposites. J. Korean Phys. Soc. 53, 376-379 (2008).

$73 \mathrm{Ma}, \mathrm{L}$., Xu, Q. \& Yang, Y. Organic nonvolatile memory by controlling the dynamic copper-ion concentration within organic layer. Appl. Phys. Lett. 84, 4908-4911 (2004).

74 Joo, W. J., Choi, T. L., Lee, J., Lee, S. K., Jung, M. S., Kim, N. \& Kim, J. M. Metal filament growth in electrically conductive polymers for nonvolatile memory application. J. Phys. Chem. B 110, 23812-23816 (2006).

75 Joo, W. J., Choi, T. L., Lee, K. H. \& Chung, Y. Study on threshold behavior of operation voltage in metal filament-based polymer memory. J. Phys. Chem. B 111, 7756-7760 (2007)

76 Baek, S., Lee, D., Kim, J., Hong, S-H., Kim, O. \& Ree, M. Novel digital nonvolatile memory devices based on semiconducting polymer thin films. Adv. Funct. Mater. 17, 2637-2644 (2007).

77 Kim, T-W., Oh, S-H., Choi, H., Wang, G., Hwang, H., Kim, D-Y. \& Lee, T. Reversible switching characteristics of polyfluorene-derivative single layer film for nonvolatile memory devices. Appl. Phys. Lett. 92, 253308-253311 (2008).
78 Choi, S., Hong, S-H., Cho, S. H., Park, S-D., Park, S-M., Kim, O. \& Ree, M. Highperformance programmable memory devices based on hyperbranched copper phthalocyanine polymer thin films. Adv. Mater. 20, 1766-1771 (2008).

79 Kim, J., Cho, S., Choi, S., Baek, S., Lee, D., Kim, O., Park, S.-M. \& Ree, M. Novel electrical properties of nanoscale thin films of a semiconducting polymer: Quantitative current-sensing AFM analysis. Langmuir 23, 9024-9030 (2007).

80 Cölle, M., Büchel, M. \& deLeeuw, D. M. Switching and filamentary conduction in nonvolatile organic memories. Org. Electron. 7, 305-312 (2006).

81 Kwan, W. L., Tseng, R. J. \& Yang, Y. Multi-layer stackable polymer memory devices. Philos. Trans. R. Soc. A-Math. Phys. Eng. Sci. 367, 4159-4167 (2009).

82 Cho, B., Yun, J-M., Song, S., Ji, Y., Kim, D-Y. \& Lee, T. Direct observation of Ag filamentary paths in organic resistive memory devices. Adv. Funct. Mater. 21, 39763981 (2011)

83 Kwan, W. L., Tseng, R. J., Wu, W., Pei, Q. \& Yang, Y. Stackable resistive memory device using photo cross-linkable copolymer. IEDM Tech. Dig. 237-240 (2007)

84 Sze, S. M. \& Ng, K. K. Physics of semiconductor devices 510-547 (WileyInterscience, New York, 2006).

85 Parker, I. D. Carrier tunneling and device characteristics in polymer light-emitting diodes. J. Appl. Phys. 75, 1656-1667 (1994).

86 Lampert, M. A. \& Mark, P. Current injection in solids 1-351 (Academic Press, New York, 1970).

87 Son, D. I., Shim, J. H., Park, D. H., Jung, J. H., Lee, J. M., Park, W. I., Kim, T. W. \& Choi, W. K. Polymer-ultrathin graphite sheet-polymer composite structured flexible nonvolatile bistable organic memory devices. Nanotechnology 22, 295203 (2011).

88 Simmons, J. G. \& Verderber, R. R. New conduction and reversible memory phenomena in thin insulating films. Proc. R. Soc. A-Math. Phys. Eng. Sci. 301, 77-102 (1967).

89 Bozano, L. D., Kean, B. W., Deline, V. R., Salem, J. R. \& Scott, J. C. Mechanism for bistability in organic memory elements. Appl. Phys. Lett. 84, 607-610 (2004).

90 Ouyang, J., Chu, C-W., Szmanda, C. R., Ma, L. \& Yang, Y. Programmable polymer thin film and non-volatile memory device. Nat. Mater. 3, 918-922 (2004).

91 Ling, Q., Song, Y., Ding, S. J., Zhu, C., Chan, D. S. H., Kwong, D-L., Kang, E-T. \& Neoh, K-G. Non volatile polymer memory device based on a novel copolymer of vinylcarbazole and complexed vinylbenzoate. Adv. Mater. 17, 455 (2005).

92 Ling, Q-D., Lim, S-L., Song, Y., Zhu, C-X., Chan, D. S-H., Kang, E-T. \& Neoh, K-G. Nonvolatile polymer memory device based on bistable electrical switching in a thin film of poly(N-vinylcarbazole) with covalently bonded $\mathrm{C}_{60}$. Langmuir 23, 312-319 (2007).

$93 \mathrm{Kim}, \mathrm{S}$. \& Lee, J. Flexible organic transistor memory devices. Nano Lett. 10, 2884-2890 (2010).

94 Moller, S., Perlov, C., Jackson, W., Taussig, C. \& Forrest, S. R. A polymer/ semiconductor write-once read-many-times memory. Nature 426, 166-169 (2003).

95 Lai, Q., Li, Z., Zhang, L., Li, X., Stickle, W. F., Zhu, Z., Gu, Z., Kamins, T. I., Williams, R. S. \& Chen, Y. An organic/Si nanowire hybrid field configurable transistor. Nano Lett. 8, 876-880 (2008).

96 Son, D., Kim, J., Park, D., Choi, W. K. Li, F., Ham, J. H. \& Kim, T. W. Nonvolatile flexible organic bistable devices fabricated utilizing CdSe/ZnS nanoparticles embedded in a conducting poly $\mathrm{N}$-vinylcarbazole polymer layer. Nanotechnology 19, 055204 (2008)

97 Tseng, Z., Kao, P., Shih, M., Huang, H., Wang, J. \& Chu, S. Electrical bistability in hybrid $\mathrm{ZnO}$ nanorod/polymethylmethacrylate heterostructures. Appl. Phys. Lett. 97, 212103 (2010).

98 Yuan, B., Hu, S., Lu, N., Xu, F., Zhou, K., Ma, Y. \& Li, M. Electrical bistability in selfassembled hybrid multilayers of phospholipid and nanoparticles. Nanotechnology 22, 315303 (2011).

99 Son, D., Park, D., le, S., Choi, W., Choi, J., Li, F. \& Kim, T. Single active-layer structured dual-function devices using hybrid polymer-quantum dots. Nanotechnology 19,395201 (2008)

$100 \mathrm{Ji}$, Y., Lee, S., Cho, B., Song, S. \& Lee, T. Flexible organic memory devices with multilayer graphene electrodes. ACS Nano 5, 5995-6000 (2011).

101 Lee, S., Kim, H., Yun, D., Rhee, S. \& Yong., K. Resistive switching characteristics of $\mathrm{ZnO}$ thin film grown on stainless steel for flexible nonvolatile memory devices. Appl. Phys. Lett. 95, 262113 (2009).

This work is licensed under the Creative Commons Attribution-NonCommercial-No Derivative Works 3.0 Unported License. To view a copy of this license, visit http:// creativecommons.org/licenses/by-nc-nd/3.0/ 


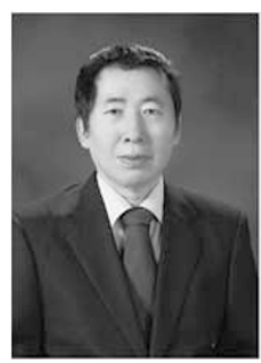

Professor Tae Whan Kim received his PhD degree in the Department of Physics from the State University of New York at Buffalo in 1989. He is a full professor at the Hanyang University in the Department of Electronic Engineering. Professor Kim's research speciality is nanodevices and nanomaterials for both investigations of fundamental physics and promising applications in electronic and optoelectronic devices. His current interests are focused on the fabrication of next-generation nonvolatile memory devices, organic light-emitting devices and solar cells.

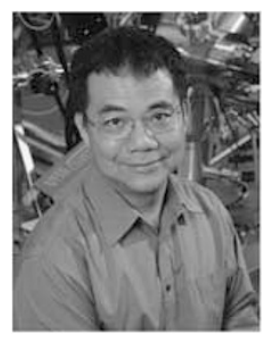

Professor Yang Yang received his PhD degree in Physics and Applied Physics from the University of Massachusetts, Lowell, in 1992. He is the Carol and Lawrence E Tannas Jr Endowed Chair Professor in the University of California, Los Angeles. Professor Yang's major researches are in the solar energy and highly efficient electronic devices, such as novel memory devices and vertical organic transistor. He has more than 250 refereed papers (including book chapters), 50 patents (filed or issued) and 120 invited talks. His H-Index is 70 as May 2012. His major contribution in the organic photovoltaics are the understanding of polymer morphology and the influence on device performance, the invention of inverted organic solar cell, inverted organic tandem solar cell, and photovoltaic polarizer for the LCDs.

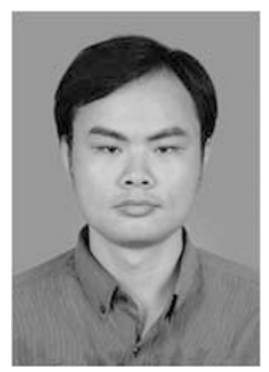

Professor Li Fushan received his PhD degree at School of Physics from the Peking University in 2005. From September 2006 to February 2009, he worked as a research professor at the Hanyang University in Korea, and from March 2009 to October 2009, he was a research fellow at the Nanyang Technological University in Singapore. Dr Li has been with School of Physics and Information Engineering of Fuzhou University as a full professor since October 2009. Dr Li's research speciality is nanoelectronic devices and fabrication technology, especially on the optoelectronic properties of various nanomaterials for application in next-generation photovoltaic, nonvolatile memory and light-emitting devices. Dr Li has published over 60 research articles in major peer-reviewed scientific journals (over 50) and conferences (over 10) and is a co-inventor of 5 International patents, 10 Korean patents and 11 Chinese patents.

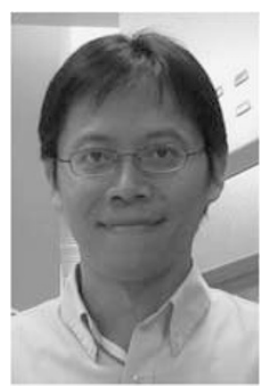

Dr Wei Lek Kwan received his PhD degree at the Department of Materials Science and Engineering from the University of California, Los Angeles, under the supervision of Professor Yang Yang in 2009. During his PhD, he worked on stackable polymer memory devices and their underlying mechanism. Dr Wei Lek Kwan is currently a lecturer at the Singapore Polytechnic and an adjunct faculty at the Singapore Institute of Technology. He currently leads a group focusing on organic photovoltaic, organic dyes and printed electronics. 